Überlegungen für Deutschland und Ansätze in Großbritannien

\title{
Ökosteuer und Zertifikate - Gegensatz oder Ergänzung?
}

\section{Bisher galt in Deutschland die ökologische Steverreform als Königsinstrument für Schritte in Richtung eines ökologischen Strukturwandels. Allerdings bleibt die praktische Umsetzung hinter diesem Anspruch zurück. International gewin- nen Emissionszertifikate an Bedeutung. Ergibt sich daraus eine neve Perspekti- ve oder handelt es sich um eine bloße Neuauflage einer Instrumentendebatte, die wirkliche Strukfurveränderungen verhindern soll?}

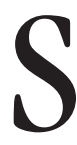

\section{$S$} eit April 1999 gibt es die Ökologische Steuerreform (ÖSR) in Deutschland. In der öffentlichen Wahrnehmung zeichnet sie sich bisher allerdings eher durch Negativ- als durch Positivschlagzeilen aus. Aufgrund des öffentlichen Streits bei der Einführung und zu großzügigen Ausnahmeregelungen für die gewerbliche Wirtschaft wurde in den letzten zwei Jahren kaum ein gutes Haar an der ÖSR gelassen. Hinzu kamen sprunghaft ansteigende Rohölpreise am Weltmarkt, die eine Akzeptanz zusätzlich erschwerten. Darüber hinaus wird - obwohl die nächsten ÖSRSchritte bis 2003 gesetzlich vereinbart sind - vor und hinter den Kulissen bereits heute heftig über eine neue Ausgestaltung diskutiert.

Vor dem Hintergrund des Kyoto-Protokolls, der UN-Klimakonferenz diesen Juli in Bonn und der "Rio+10"-Konferenz im September 2002 in Johannesburg wird dagegen verstärkt ein neues Instrument diskutiert, das insbesondere seitens der Wirtschaft als Ersatz für die ÖSR gehandelt wird: der Handel mit Emissionszertifikaten. Schließen sich beide Maßnahmen aus oder ist ein sinnvolles Zusammenspiel anzustreben, um den ökologischen Strukturwandel, die Reduzierung der Treibhausgase und eine effiziente Ressourcennutzung voranzutreiben?

Sowohl die ÖSR als auch der Zertifikatehandel sind marktwirtschaftliche Instrumente staatlicher Umweltpolitik. Die ÖSR versucht, die Nutzung fossiler Ressourcen und die Kohlendioxidemissionen über allmählich aber stetig steigende Steuersätze - und damit über den Preis - zu verringern. Die Menge an emittierten Treibhausgasen ist jedoch von der Reaktion der Marktteilnehmer auf die steigenden Preise abhängig.
Der Handel mit Emissionszertifikaten geht genau den umgekehrten Weg. Sind vom Staat erst einmal Zertifikate an die Marktteilnehmer verteilt, kann diese Menge vom Staat - oder von einer zuständigen Aufsichtsbehörde - in stetigen, planbaren Schritten verringert werden. Die Marktteilnehmer wären dann zur Einsparung von Emissionen gezwungen oder müssten sich bei unveränderten oder steigenden Treibhausgasemissionen über den Markt von anderen - progressiveren - Teilnehmern zusätzliche Zertifikate kaufen. Das Klimaschutzziel könnte mit dem Emissionshandel im Gegensatz zur ÖSR - auf den Punkt genau erreicht werden. Unklar wäre aber der Preis der Zertifikate, der nicht vorgegeben wird, sondern sich am Markt bildet. Dies ist eine der größten Unsicherheiten des Zertifikatehandels.

Das zweite bisher noch ungelöste Problem des Zertifikatehandels ist die Anzahl der Marktteilnehmer. Der Handel funktioniert nur dann, wenn es nicht zu viele Marktteilnehmer gibt und am Markt eine größtmögliche Transparenz herrscht. So funktioniert ein Schwefeldioxid-Zertifikatehandel zwischen wenigen industriellen Emittenten in den USA seit Jahren sehr gut; auch große Konzerne wie BP haben konzernintern einen funktionierenden Zertifikatehandel aufgebaut. Gänzlich ungelöst ist allerdings bisher das Problem, wie ein Handel in Deutschland mit über 80 Millionen Marktteilnehmern oder gar ein länderübergreifender Handel organisiert werden könnte.

\section{Vorteile beider Instrumente nutzen}

An Stelle eines drohenden Konkurrenzkampfes zwischen ÖSR und Zertifikatehandel könnte in Zukunft ein abgestimmtes Konzept aus beiden Maßnahmen stehen
ÖSR und Zertifikate ergänzen sich mit ihren jeweiligen Stärken und Schwächen sehr gut. Die ÖSR entfaltet über steigende Mineralöl- und Stromsteuern besonders in den Bereichen Verkehr und private Haushalte ihre Wirkung - Bereiche, in denen die Praktikabilität von Zertifikaten bisher noch nicht nachgewiesen werden konnte. Der Zertifikatehandel könnte dagegen insbesondere im Bereich der gewerblichen Wirtschaft ein probates Mittel zur Reduktion von Treibhausgasen darstellen.

Bei der Debatte um die Fortführung und zukünftige Ausgestaltung der ÖSR wäre vor diesem Hintergrund eine beengte Sicht auf die Reduzierung der derzeit bestehenden - und zweifelsohne zu weit reichenden - Ausnahmeregelungen für die gewerbliche Wirtschaft zu kurz gegriffen (1).

Mit dem Beschluss des Bundeskabinetts im 0ktober 2000 zum nationalen Klimaschutzprogramm (2) wurde eine Arbeitsgruppe Emissionshandel zur Bekämpfung des Treibhauseffektes eingesetzt. In dieser diskutieren unter Federführung des Bundesumweltministeriums (BMU) Vertreter verschiedener Bundesministerien mit Vertretern von Wirtschaft und Umweltverbänden über Eckpunkte für ein Emissionshandelskonzept (3). Im Grünbuch der EU-Kommission zum Emissionshandel (4) wird die Zulassung eines Emissionshandels innerhalb von Mitgliedstaaten ab 2005 und ein zwischenstaatlicher Handel ab 2008 angestrebt. Ein darauf aufbauender Richtlinienentwurf der Kommission für die Umsetzung in den EU-Mitgliedstaaten ist in Vorbereitung.

Überlegungen über die zukünftige Ausgestaltung der ÖSR in Deutschland sollten daher einen möglichen Zertifikatehandel einbeziehen. Denkbar wäre beispielsweise die Abschaffung des bisher geltenden "Spitzenbelastungsausgleichs" in der ÖSR. Durch diesen bekommen Unternehmen den Anteil ihrer Energiesteuerzahlungen, der das 1,2fache der Rückerstattung durch sinkende Rentenversicherungsbeiträge übersteigt, auf Antrag erstattet. Statt dessen könnten diese Unternehmen an einem nationalen Kohlendioxid-Zertifikatehandel teilnehmen.

Der Parteirat von Bündnis 90/Die Grünen geht in seinem jüngsten Beschluss zur ÖSR (5) sogar noch einen Schritt weiter. Er fordert eine deutsche Führungsrolle in der internationalen Debatte um den Emissionshandel und bezeichnet die Einführung eines Emissionshandelssystems mit absoluten Emissionsobergrenzen als Ersatz für die "Ökosteuer für die Industrie" ab 2005 als mögliche Option. 


\section{- Auf Emissionshandel vorbereitet sein}

Der bei BP seit Anfang 2000 zwischen 150 Geschäftseinheiten des Konzerns weltweit funktionierende Handel mit Emissionszertifikaten (für einen Mix der Treibhausgase Kohlendioxid und Methan) zeigt auf beeindruckende Weise die Effizienz des Modells: Der Preis für die derzeit gehandelten Emissionslizenzen lag nach BP-Aussagen im Frühjahr 2001 mit durchschnittlich 7,5 US-Dollar pro Tonne deutlich unter den zunächst erwarteten Kosten. Durch den Emissionshandel bekommt die Emission von Treibhausgasen erstmals einen real begreifbaren Preis, der sich mit Investitionen in die Energieeffizienz abgleichen lässt.

Deutschland sollte nicht darauf warten, dass in übergeordneten Institutionen wie der EU oder auch bei den nächsten UN-Klimakonferenzen über einen Emissionshandel entschieden wird. Durch das frühzeitige Sammeln eigener Erfahrungen mit einem integrierten Konzept aus ÖSR und Emissionshandel hätte Deutschland die Chance, im internationalen Klimaschutz Wettbewerbsvorteile zu erlangen - bei Wahrung einer größtmöglichen Flexibilität der eingesetzten Instrumente. Großbritannien ist auf einem solchen Weg bereits einen Schritt weiter (vgl. den Kasten).

Zumindest national besteht jedoch auch die Gefahr, dass ÖSR und Emissionshandel in der Debatte um die Fortführung der Ökologischen Steuerreform und Klimaschutz gegeneinander ausgespielt werden. Dieser Debatte kann durch ein integriertes Konzept schon im Vorfeld die Basis entzogen werden. Da sich allerdings bereits jetzt in Teilen der Industrie der Widerstand gegen einen Emissionshandel formiert, sind auch bei diesem Instrument ähnliche zähe und kontroverse Diskussionen wie bei der Einführung der ÖSR zu erwarten.

Analog zur ÖSR sind beim Emissionshandel noch zahlreiche Probleme zu lösen, wie zum Beispiel die Abgrenzung von energieintensiven Branchen und die Auswirkungen auf die Wettbewerbsfähigkeit. Die Rahmenbedingungen für den Emissionshandel - wie etwa Zuteilung, Reduktionsziel, Sanktionen - müssen daher vom Gesetzgeber vor Einführung des Handels klar definiert werden. Daher sollten frühzeitig - möglichst schon heute - Modelle und Planspiele zur Umsetzung entwikkelt werden.

Die Diskussion um Emissionszertifikate darf aber - bei stets wachsenden Ausnahmen von der ÖSR in Form steigender Subventionen (9) - nicht

\section{Zusammenspiel von Climate Change Levy und Emissionshandel in Großbritannien}

Vom britischen Finanzminister wurde nach der Klimakonferenz in Kyoto 1997 eine Projektgruppe eingesetzt, die untersuchen sollte, wie Treibhausgasemissionen reduziert werden können ohne dabei die Wettbewerbsfähigkeit der heimischen Industrie zu beeinträchtigen. Im Ende 1998 vorgelegten Abschlußbericht (6) wurden Maßnahmen wie der Zertifikatehandel und Energiesteuern fokussiert, wobei der Gebrauch von Zertifikaten als Langzeitstrategie gesehen wurde, die mit praktischen Schwierigkeiten verbunden sei und deshalb zum damaligen Zeitpunkt (noch) nicht für sinnvoll erachtet wurde. Die Einführung einer Energiesteuer (Climate Change Levy - $\mathrm{CCL}$ ) erschien als leichter umsetzbar und wurde nach intensiven Beratungen mit Energieunternehmen, gewerblichen Nutzern, Umweltorganisationen und Wissenschaftlern in einem offenen Prozess angekündigt - parallel zur Einführung der ÖSR in Deutschland im Frühjahr 1999.

Am 1. April 2001 wurde die CCL in Großbritannien mit dem in Kyoto gesetzten Ziel einer Verringerung der Treibhausgasemissionen um 12,5 Prozent im Vergleich

dazu benutzt werden, eine erneute Instrumentendiskussion zu führen, ohne eine konkrete Umsetzung als Ziel zu haben. Immerhin hat es bereits drei fehlgeschlagene Anläufe zur Einführung eines Zertifikatehandels seitens des BMU in Deutschland gegeben - die Kraft-Wärme-Kopplung ist hier das aktuellste Beispiel.

Liegen die Ursachen für diese bisher gewonnenen negativen Erfahrungen im fehlenden Leistungsvermögen des Systems oder in der fehlenden Bereitschaft der deutschen Wirtschaft, derartige Ansätze konstruktiv aufzugreifen (10)? Die für das Jahresende 2001 angekündigten Ergebnisse der Emissionshandels-Arbeitsgruppe, an der die Wirtschaft maßgeblich beteiligt ist, werden eine erste Antwort auf diese Frage geben. Gleichzeitig dienen sie als Grundlage für eine Diskussion über das $O b$ und Wie der Implementierung eines Emissionshandelssystems und das Zusammenwirken mit der ÖSR.

\section{Anmerkungen}

(1) Vgl. zur Reformdiskussion Schrader, B./Schlegelmilch, K.: Die Ökosteuer ist besser als ihr Ruf aber sie kann noch besser werden. In: VÖW (Hrsg.): Ökologische Modernisierung. Berlin 2001, S. 10-12, sowie den Beitrag zu den ÖSR-Sonderregelungen von B. Meyer in Ökologisches Wirtschaften, Nr 3-4/2000, S. 6-7.

(2) Internet-Download unter http://www.bmu.de.

(3) Die AG will nach eigenen Aussagen ab Sommer 2001

über die Homepage des Bundesumweltministeriums

(http://www.bmu.de) regelmäßig über den Diskussions-
zU 1990 eingeführt. Im Gegensatz zur ÖSR richtet sich die Steuer nur auf den industriellen Energieverbrauch. Energieintensiven Unternehmen, die von der EU-IVURichtlinie zur integrierten Vermeidung und Verminderung von Umweltbelastungen erfasst werden, wird eine 80-prozentige Reduzierung der Steuer gewährt, wenn sie alle Energiesparmaßnahmen tätigen, die auch kosteneffizient sind (7).

Eine Möglichkeit für Unternehmen, in den Genuss dieser Steuerreduktion zu kommen, ist die Teilnahme an einem freiwilligen nationalen Kohlendioxid-Emissionshandel, der sich derzeit im Aufbau befindet. Die im Juni 1999 gegründete Emissions Trading Group (ETG) hat hierfür ein Modell entwickelt, das vor kurzem veröffentlicht wurde (8). Britischen Unternehmen eröffnet sich dadurch die Option, an einem möglichen weltweiten Emissionshandelssystem nach Artikel 17 des Kyoto-Protokolls teilzunehmen, für das sie durch das nationale Handelssystem wertvolle Erfahrungen sammeln können.

stand und erzielte Fortschritte berichten.

(4) Grünbuch zum Handel mit Treibhausgasemissionen in der Europäischen Union - KOM (2000)87 endg. vom 8.3.2000.

(5) Beschluss des Parteirats von Bündnis 90/Die Grünen vom 2.7.2001: Ökologisch Steuern für den Klimaschutz Deutschlands internationale Verantwortung wahrnehmen. (6) HM Treasury: Economic Instruments and the Business Use of Energy. A Report by Lord Marshall. November 1998. (7) HM Treasury: Stability and Steady Growth for Britain. Pre-Budget Report, November 1999.

(8) Im Internet unter http://www.defra.gov.uk/environment/climatechange/trading/pdf/trading-full.pdf.

(9) Vgl. den 18. Subventionsbericht der Bundesregierung, der am 25.07.2001 vom Bundeskabinett beschlossen wurde (BT-Drs. 14/6748).

(10) Schafhausen, F.: Zertifikatsregelung - Konzeptionelle Schwierigkeiten, instrumentelles Leistungsvermögen, erforderliche Rahmenbedingungen. In: AGU/ DłA (Hrsg.): Umweltforum `99, Bonn 1999, S. 35.

\section{Die Autorlnnen}

Mario Meinecke ist wissenschaftlicher Mitarbeiter im Büro von Dr. Ernst U. von Weizsäcker, MdB. Kontakt: Tel. 030/ 227-74713, Fax -76721, E-mail: Mario.Meinecke@gmx.de Bettina Schrader ist wissenschaftliche Mitarbeiterin an der Universität Bremen und VÖW-Vorstand. Kontakt: Tel. 0421/ 218-9118, Fax -7007, E-mail: bettina.schrader@uni-bremen.de 
(c) 20I0 Authors; licensee IÖW and oekom verlag. This is an article distributed under the terms of the Creative Commons Attribution Non-Commercial No Derivates License (http://creativecommons.org/licenses/by-nc-nd/3.o/), which permits unrestricted use, distribution, and reproduction in any medium, provided the original work is properly cited. 